JAIST Repository

https://dspace.jaist.ac.jp/

\begin{tabular}{|c|c|}
\hline Title & On const ruct ing compl et i ons \\
\hline Author(s) & $\begin{array}{l}\text { Crosi Il a, Laur a; I shi har a, Haj i me; Schuster, } \\
\text { Pet er }\end{array}$ \\
\hline Citation & The Journal of Symbol i c Logi c, 70(3): 969-978 \\
\hline Issue Date & 2005- 09 \\
\hline Type & Jour nal Arti cle \\
\hline Text version & publ i sher \\
\hline URL & ht t p: //hdl . handl e. net /10119/8527 \\
\hline Rights & $\begin{array}{l}\text { Copyri ght ( C) } 2005 \text { Associ at i on f or Symbol i c } \\
\text { Logi c. It is post ed here by per mi ssi on of } \\
\text { Associ at i on f or Symbol i c Logi c. Laur a Cr osi I I a, } \\
\text { Haj i me I shi har a, and Pet er Schust er, The Jour nal } \\
\text { of Symbol i c Logi c, } 70 \text { (3), 2005, 969-978. }\end{array}$ \\
\hline Desc & \\
\hline
\end{tabular}

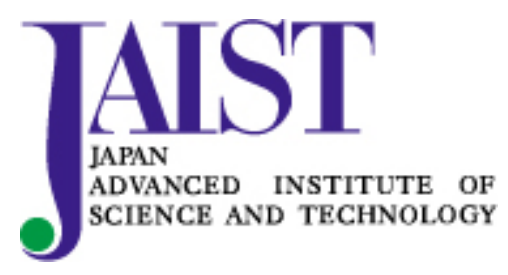




\title{
ON CONSTRUCTING COMPLETIONS
}

\author{
LAURA CROSILLA, HAJIME ISHIHARA, AND PETER SCHUSTER
}

\begin{abstract}
The Dedekind cuts in an ordered set form a set in the sense of constructive Zermelo-Fraenkel set theory. We deduce this statement from the principle of refinement, which we distill before from the axiom of fullness. Together with exponentiation, refinement is equivalent to fullness. None of the defining properties of an ordering is needed, and only refinement for two-element coverings is used.

In particular, the Dedekind reals form a set; whence we have also refined an earlier result by Aczel and Rathjen, who invoked the full form of fullness. To further generalise this, we look at Richman's method to complete an arbitrary metric space without sequences, which he designed to avoid countable choice. The completion of a separable metric space turns out to be a set even if the original space is a proper class; in particular, every complete separable metric space automatically is a set.
\end{abstract}

§1. Introduction. Throughout this article we work in the constructive ZermeloFraenkel set theory (CZF) founded by Aczel in [2] as a formal system for Bishop's constructive mathematics [7, 8]. CZF came out two years after Myhill's constructive set theory (CST) [18], and was further developed in [3, 4]. More recently, Rathjen and others have joined Aczel in the enterprise of CZF; we refer to [6] also for parts of this later progress. The main characteristics of CZF can be summarised as follows.

On the one side, CZF is formulated in the same language as classical ZermeloFraenkel set theory $(\mathrm{ZF})$ is, and in such a way that full ZF results from it by simply adding the logical principle of excluded middle. On the other side, CZF takes advantage of its being based on intuitionistic logic, as it avoids the classical axioms of full separation and of power set in favour of (intuitionistically) weaker principlesrestricted separation and subset collection, respectively — which can be justified from a tolerant predicative perspective. As a consequence, the proof-theoretic strength of $\mathrm{CZF}$ is well below the one of ZF, contrary to impredicative systems like intuitionistic Zermelo-Fraenkel set theory (IZF). Finally, while introducing his system Aczel has also provided it with a natural interpretation in Martin-Löf's intuitionistic type theory (ITT) [15, 16, 17], by means of which CZF gains constructive meaning as well as a computational content for its theorems.

In the present note we study whether one obtains sets in the sense of CZF when one constructs completions - and, if so, which principles of CZF are needed to ensure this. We start with the real numbers, perhaps the most basic completion occurring in mathematics. With Dedekind reals one can follow the standard development of constructive analysis also in an environment, such as topos theory, in which countable choice cannot be taken for granted. The situation is different for Cauchy

Received July 22, 2004; accepted April 1, 2005. 
reals, which one has to equip throughout with moduli of convergence etc. to avoid countable choice.

Also when it comes to show that the class of real numbers is a set, then Cauchy and Dedekind reals have to be treated differently. The Cauchy reals form a set by virtue of the principle of exponentiation, which is an axiom of CST, and valid in $\mathrm{CZF}$ as a consequence of subset collection. In CST, moreover, dependent choice is included; whence the Dedekind reals coincide with the Cauchy reals, and thus form a set. This rather simple argument does not work in CZF, in which theory not even countable choice is at one's disposal.

However, Aczel and Rathjen have deduced from the principle of fullness - an equivalent of subset collection - that the Dedekind reals form a set in CZF [6]. As we shall see below, to prove this it suffices to assume a binary form of fullness. We also show that, more generally, Richman's choice-free way to complete a separable metric space [21] yields a completion which is a set no matter whether the original space is a set.

The consequence of fullness we start from is that for every pair of sets $A$ and $B$ there is a set $D$ (of subsets of $A$ ) such that every covering of $A$ by a family of subsets with index set $B$ admits of a refinement whose elements all belong to $D$. Accordingly, we call this the principle of refinement; we prove that in conjunction with exponentiation it is equivalent to fullness. Given refinement for $A$ and $B$, it suffices that $D^{B}$ is a set to deduce the corresponding instance of fullness.

To deal with completions, we only need the specific case of refinement in which $B$ is the two-element set. This, and more generally refinement for finite coverings, turns out to be equivalent - even without exponentiation - to the corresponding instance of fullness. Refinement has nonetheless the advantage of a topological character, for which it is probably easier to use than fullness, especially in mathematics beyond set theory. The appearance of the principle of refinement may thus help to represent formal topology in CZF, which task was put forward in [6] and begun in [1, 5].

§2. The axioms of CZF. The language of CZF is the first-order language of Zermelo-Fraenkel set theory with the usual non-logical primitive symbols $\in$ and $=$. The logical symbols are all the intuitionistic operators $\perp, \wedge, \vee, \rightarrow, \exists$, and $\forall$; in particular, $\neg \varphi$ is defined as $\varphi \rightarrow \perp$. In addition, $\exists x \in y \varphi$ and $\forall x \in y \varphi$ stand for $\exists x(x \in y \wedge \varphi)$ and $\forall x(x \in y \rightarrow \varphi)$, respectively. As usual, $x \subseteq y$ is a shorthand for $\forall z \in x(z \in y)$.

A formula of $\mathrm{CZF}$ is restricted, or bounded or a $\Delta_{0}$-formula, if all quantifiers occurring in it -if any-are bounded: that is, they are of the form $\exists x \in y$ or $\forall x \in y$. The axioms of CZF are the usual axioms for intuitionistic first-order logic with equality together with the following eight set-theoretic axioms and axiom schemes.

\section{Extensionality}

$$
\forall a \forall b(\forall y(y \in a \leftrightarrow y \in b) \rightarrow a=b) .
$$

\section{Pair}

$$
\forall a \forall b \exists x \forall y(y \in x \leftrightarrow y=a \vee y=b)
$$




\section{Union}

$$
\forall a \exists x \forall y(y \in x \leftrightarrow \exists z \in a y \in z) .
$$

4. Restricted Separation For every $\Delta_{0}$-formula $\varphi(y)$,

$$
\forall a \exists x \forall y(y \in x \leftrightarrow y \in a \wedge \varphi(y)) .
$$

5. Subset Collection For every formula $\varphi(x, y, u)$,

$$
\begin{aligned}
& \forall a \forall b \exists c \forall u(\forall x \in a \exists y \in b \varphi(x, y, u) \\
& \rightarrow \exists d \in c(\forall x \in a \exists y \in d \varphi(x, y, u) \wedge \forall y \in d \exists x \in a \varphi(x, y, u))) .
\end{aligned}
$$

6. Strong Collection For every formula $\varphi(x, y)$,

$\forall a(\forall x \in a \exists y \varphi(x, y)$

$$
\rightarrow \exists b(\forall x \in a \exists y \in b \varphi(x, y) \wedge \forall y \in b \exists x \in a \varphi(x, y))) .
$$

\section{Strong Infinity}

$$
\begin{aligned}
& \exists x((0 \in x \wedge \forall y(y \in x \rightarrow y+1 \in x) \\
& \wedge \forall z(0 \in z \wedge \forall y(y \in z \rightarrow y+1 \in z) \rightarrow x \subseteq z)),
\end{aligned}
$$

where as usual $y+1$ is the successor $y \cup\{y\}$ of $y$, and 0 is the empty set $\emptyset$.

8. Set Induction For every formula $\varphi(a)$,

$$
\forall a(\forall x \in a \varphi(x) \rightarrow \varphi(a)) \rightarrow \forall a \varphi(a) .
$$

Principles 1-6, and 8 are the same as in [2]. We only diverge from that paper inasmuch as we adopt the axiom of infinity in a slightly stronger formulation which guarentees the existence of a least inductive set. This uniquely determined set of natural numbers will be denoted by $\mathbb{N}$.

With regard to the other axioms of $\mathrm{ZF}$, we recall that in the presence of intuitionistic logic one needs to use set induction in place of the axiom of foundation, since the latter would entail the principle of excluded middle for $\Delta_{0}$-formulas. In addition, full separation and power set are replaced by restricted separation and subset collection, respectively, to conform to a notion of predicativity. ${ }^{1}$ Strong collection is taken in place of collection for technical reasons, due to the restriction on separation.

An obvious consequence of strong collection is the following principle.

Replacement: For every formula $\varphi(x, y)$,

$$
\forall a(\forall x \in a \exists ! y \varphi(x, y) \rightarrow \exists b \forall y(y \in b \leftrightarrow \exists x \in a \varphi(x, y))) .
$$

One can rephrase it in the perhaps pithier way that if the domain of a mapping is a set, then so is its range. In particular, the principle of replacement together with the axiom of (strong) infinity ensures that every countable class is a set.

\footnotetext{
${ }^{1}$ In $\mathrm{CZF}$ we form, by separation, a subset of a given set only if the quantifiers in the formula defining the new set are bounded, and so only refer to previously constructed sets; in particular, we do not want them to refer to the totality of sets. The axiom of power set does not conform to predicativity, as it allows to form a set of all subsets of a given (possibly infinite) set, without providing any information on how such a collection should be formed.
} 
$\S 3$. The principle of refinement. Let $A$ and $B$ be sets. By repeated use of replacement, one can introduce a set $A \times B$, the cartesian product of $A$ and $B$, which consists of the ordered pairs $(x, y)=\{\{x\},\{x, y\}\}$ with $x \in A$ and $y \in B$. A relation $r$ between $A$ and $B$ is a subset of $A \times B$; it is total whenever for every $x \in A$ there is $y \in B$ with $(x, y) \in r$. A (total) relation $r \subseteq A \times B$ is a function from $A$ to $B$ if for every $x \in A$ there is exactly one $y \in B$ with $(x, y) \in r$. As total relations can be seen as multivalued functions, the class of total relations from $A$ to $B$ is often denoted by $\operatorname{mv}(A, B)$. One cannot assume in general that the class $\mathrm{mv}(A, B)$ is a set, as this would amount to include power set.

On the basis of the remaining principles of CZF (in particular, of strong collection), subset collection is equivalent to the following.

Fullness: There is a set $C$ with $C \subseteq \operatorname{mv}(A, B)$ such that for every $r \in \operatorname{mv}(A, B)$ there is $s \in C$ with $s \subseteq r$.

We use the symbol $B^{A}$ for the class of functions from $A$ to $B$. The next principle of CZF follows from the foregoing.

Exponentiation: $B^{A}$ is a set.

In fact, $B^{A}$ can be separated from a set $C$ as provided by fullness, because to be a function is a restricted property of a (total) relation. ${ }^{2}$

By restricted separation, for each relation $r \subseteq A \times B$ and every $b \in B$ the fibre

$$
r^{-1}(b)=\{a \in A:(a, b) \in r\}
$$

of $r$ over $b$ is a subset of $A$. The following variant of fullness seems to be new.

Refinement: There is a set $D$ (of subsets of $A$ ) such that for every $r \in \operatorname{mv}(A, B)$ there is $s \in \operatorname{mv}(A, B)$ with $s \subseteq r$ such that $s^{-1}(b) \in D$ for all $b \in B$.

The reason why we have chosen the name of the principle of refinement will become clear only later.

THeOREm 3.1. Fullness implies refinement. Conversely, fullness for $A$ and $B$ follows from the same instance of refinement together with the condition that $D^{B}$ is a set.

Proof. Let $C$ be as in fullness, and define

$$
D=\left\{s^{-1}(b): s \in C \& b \in B\right\} .
$$

This $D$ is a set (of subsets of $A$ ) by replacement. According to our choice of $C$, for every $r \in \operatorname{mv}(A, B)$ there is $s \in C$ with $s \subseteq r$. For any such $s$, clearly, $s^{-1}(b) \in D$ for all $b \in B$. In other words, $D$ is as required in refinement.

Conversely, let $D$ be as in refinement with the additional property that $D^{B}$ is a set. Define

$$
C^{\prime}=\left\{s \subseteq A \times B: \forall b \in B\left(s^{-1}(b) \in D\right)\right\}
$$

and

$$
C=C^{\prime} \cap \operatorname{mv}(A, B) .
$$

Although $C$ is a priori only a class, if $r \in \operatorname{mv}(A, B)$, then - by our choice of $D$ there is $s \in C$ with $s \subseteq r$. Hence $C$ fulfils the requirements of fullness once we have shown that it is a set, with which we conclude this proof.

\footnotetext{
${ }^{2}$ Lubarsky has recently shown that exponentiation does not prove subset collection [14].
} 
Since to be total is a restricted property of relations between $A$ and $B$, we only need to show that $C^{\prime}$ is a set. To this end, consider for each $f \in D^{B}$ the relation $r_{f} \subseteq A \times B$ with

$$
r_{f}=\bigcup\{f(b) \times\{b\}: b \in B\},
$$

for which $r_{f}^{-1}(b)=f(b) \in D$ for every $b \in B$, and thus $r_{f} \in C^{\prime}$. In other words, $C^{\prime \prime} \subseteq C^{\prime}$ with

$$
C^{\prime \prime}=\left\{r_{f}: f \in D^{B}\right\} .
$$

However, we even have $C^{\prime}=C^{\prime \prime}$, because if $s \in C^{\prime}$, then $s=r_{f}$ with $f \in D^{B}$ defined by setting $f(b)=s^{-1}(b)$ for every $b \in B$. By replacement, $C^{\prime \prime}$ is a set, and so is $C^{\prime}$.

As exponentiation is a consequence of fullness, we furthermore have the following.

Corollary 3.2. Fullness is equivalent to refinement together with exponentiation.

If $B$ is an $n$-element set for some $n \geqslant 1$, then there is a bijection between $D^{B}$ and the $n$-th cartesian power $D^{n}$ of $D$; whence $D^{B}$ is a set without exponentiation. All finite cartesian powers of a set are indeed sets, by induction over the natural numbers, which proof principle for arbitrary formulas is a consequence of set induction.

Corollary 3.3. Fullness for $A$ and any $n$-element set $B$ is equivalent to the same instance of refinement.

Let $U$ be a family of subsets of $A$ indexed by $B$ : that is, for every $b \in B$ there is a uniquely determined subset $U(b)$ of $A$. In this case, $\{U(b): b \in B\}$ is a set by replacement. If $A=\bigcup\{U(b): b \in B\}$, then we call $U$ a covering family or simply a covering of $A$ indexed by $B$. By a refinement of a covering $U$ we understand a covering $V$ with $V(b) \subseteq U(b)$ for every $b \in B$.

REMARK 3.4. If $r \subseteq A \times B$ is a total relation, then $U_{r}$ with

$$
U_{r}(b)=r^{-1}(b) \quad(b \in B)
$$

is a covering family. Conversely, if $U$ is a covering family, then

$$
r_{U}=\{(a, b) \in A \times B: a \in U(b)\}
$$

is a total relation. These two constructions are inverse to each other, and $s \subseteq r$ holds for $r, s \in \mathrm{mv}(A, B)$ precisely when $U_{s}$ is a refinement of $U_{r}$.

In view of this one-to-one correspondence between total relations between $A$ and $B$ and coverings of $A$ indexed by $B$, fullness for $A$ and $B$ is equivalent to the existence of a set $C$ of coverings of $A$, all indexed by $B$, which contains as an element a refinement of each covering of $A$ indexed by $B$. More useful than this is the following interpretation of the principle of refinement in the language of coverings, which also justifies our choice of its name.

Corollary 3.5. The principle of refinement for $A$ and $B$ is equivalent to the existence of a set $D$ (of subsets of $A$ ) such that every covering of $A$ indexed by $B$ allows for a refinement $V$ with $V(b) \in D$ for all $b \in B$. 
From the hindsight, the condition occurring in Theorem 3.1 that $D^{B}$ is a set appears to be fairly natural - every covering $V$ of $A$ indexed by $B$ with $V(b) \in D$ for all $b \in B$ is an element of $D^{B}$.

According to Corollary 3.3, fullness for $A$ and any $n$-element set $B$ is equivalent to the following, which is trivially valid for $n=1$ with $D=\{A\}$.

$n$-ary Refinement: There is a set $D$ (of subsets of $A$ ) such that if $U_{1}, \ldots, U_{n}$ are subsets of $A$ with $A=U_{1} \cup \cdots \cup U_{n}$, then there are $V_{1}, \ldots, V_{n} \in D$ with $A=V_{1} \cup \cdots \cup V_{n}$ such that $V_{i} \subseteq U_{i}$ for all $i \in\{1, \ldots, n\}$.

When it next comes to constructing completions, we will only need the case $n=2$.

Binary Refinement: There is a set $D$ (of subsets of $A$ ) such that if $G$ and $H$ are subsets of $A$ with $A=G \cup H$, then there are $V, W \in D$ with $V \subseteq G, W \subseteq H$, and $A=V \cup W$.

Again, binary refinement is equivalent to fullness for $A$ and any two-element set $B$.

$\S 4$. Order completions. Let $X$ be a set with a binary relation $<$. Although we do not require any further property from $X$ and <, we adopt the terminology of (strict) order relations. In this vein, a pair $\xi=(L, U)$ of subsets $L, U$ of $X$ is called

- disjoint if $L$ and $U$ are disjoint,

- bounded if $L$ and $U$ are inhabited,

- located if either $x \in L$ or $y \in U$ whenever $x<y$, and

- open if for every $x \in L$ there is $y \in L$ with $x<y$, and for every $y \in U$ there is $x \in U$ with $x<y$.

If $\xi$ satisfies all these four properties, then it is said to be a Dedekind cut in $X$. Every disjoint and located $\xi$ is also monotone with respect to $<$ : that is, if $x<y$ and $y \in L$, then also $x \in L$, and if $x<y$ and $x \in U$, then also $y \in U$. The 'weak' relation $\leqslant$ derived from the 'strict' relation $<$ can be defined by setting $x \leqslant y$ precisely when $\neg(y<x)$. If $\xi$ is disjoint, then $x<y$ for all $x \in L$ and $y \in U$ provided that $\xi$ is monotone with respect to $\leqslant$.

If $<$ is a pseudo-ordering in the sense of Aczel, which is the constructively meaningful concept of a strict ordering, then the Dedekind cuts form the order completion of $X$. Palmgren [20] has shown, with well-founded dependent choice, that this is a set in the sense of Martin-Löf type theory with regular universes. In CZF, Aczel and Rathjen [6] have deduced from fullness that the order completion of $\mathbb{Q}$ is a set, which is nothing but the set of Dedekind reals.

We now provide a more general argument. Apart from the fact that we use apparently weaker principles, our approach differs from the aforementioned ones inasmuch as we do not need any property of $X$ and $<$. In other words, $<$ may be an arbitrary binary relation on the set $X$.

THEOREM 4.1. Binary refinement for

$$
A=\{(x, y) \in X \times X: x<y\}
$$

implies that the disjoint, open, and located pairs of subsets of $X$ form a set.

Proof. Let $D$ be as in this instance of binary refinement, and set

$$
P=\{(V, W) \in D \times D: A=V \cup W\} .
$$


For every $\pi=(V, W) \in P$ define $\xi_{\pi}=\left(L_{\pi}, U_{\pi}\right)$ with

$$
\begin{aligned}
& L_{\pi}=\{x \in X: \exists y \in X((x, y) \in V)\}, \\
& U_{\pi}=\{y \in X: \exists x \in X((x, y) \in W)\},
\end{aligned}
$$

which are sets by restricted separation. Moreover, $\xi_{\pi}$ is a located pair for every $\pi=(V, W) \in P$, because if $(x, y) \in A$, then either $(x, y) \in V$ or $(x, y) \in W$.

Conversely, when $\xi=(L, U)$ is a located pair of subsets of $X$, then there is $\pi \in P$ with $\xi=\xi_{\pi}$ provided that, in addition, $\xi$ is disjoint and open. Indeed, if we define

$$
\begin{aligned}
& G=\{(x, y) \in A: x \in L\}, \\
& H=\{(x, y) \in A: y \in U\},
\end{aligned}
$$

then $A=G \cup H$, because $\xi$ is located. By our choice of $D$, there are $V, W \in D$ with $V \subseteq G, W \subseteq H$, and $A=V \cup W$; in particular, $\pi=(V, W) \in P$. To see that $L=L_{\pi}$ for this $\pi$, let first $x \in L_{\pi}$ : that is, $(x, y) \in V$ for some $y \in X$. Since $V \subseteq G$, we have $x \in L$. Assume next that $x \in L$. Since $\xi$ is open, there is $y \in L$ with $x<y$. Since $A=V \cup W$, we either have $(x, y) \in V$ or $(x, y) \in W$. In the former case, $x \in L_{\pi}$ as required; in the latter case, $(x, y) \in H$ and thus $y \in U$ in contradiction to $\xi$ being disjoint. To show that $U=U_{\pi}$ for this $\pi$ is completely analogous.

By restricted separation, $P$ is a set, and so is

$$
P^{\prime}=\left\{\pi \in P: \xi_{\pi} \text { is disjoint and open }\right\} .
$$

By the above, the disjoint, open, and located pairs of subsets of $X$ are precisely the elements of $\left\{\xi_{\pi}: \pi \in P^{\prime}\right\}$, which is a set by replacement.

We can separate the Dedekind cuts from the disjoint, open, and located pairs of subsets of $X$, because boundedness is a restricted property.

Corollary 4.2. By binary refinement, the Dedekind cuts in $X$ form a set.

A particular consequence of binary refinement is therefore that the Dedekind cuts in $\mathbb{Q}$ with the usual ordering form a set, the set $\mathbb{R}$ of Dedekind reals. These are isomorphic to the formal reals [19], the real numbers in formal topology [22], and to define this isomorphism requires restricted separation only. Hence binary refinement implies, by Corollary 4.2, that the formal reals form a set once they are carried over to $\mathrm{CZF}$.

§5. Metric completions. To avoid countable choice, Richman [21] has developed a method to complete an arbitrary metric space $S$ with no mention of sequences. We now briefly recall his approach, including the sequence-free variants of some topological concepts.

In our context, a metric space is a class $S$ endowed with a mapping $d: S \times S \rightarrow \mathbb{R}$ satisfying the usual three conditions

$$
\begin{aligned}
& d(x, y)=0 \Leftrightarrow x=y \\
& d(x, y)=d(y, x) \\
& d(x, z) \leqslant d(x, y)+d(y, z)
\end{aligned}
$$

for all $x, y, z \in S$. If $S$ is a metric space and $T \subseteq S$, then the closure $\bar{T} \subseteq S$ of $T$ consists of all the $x \in S$ such that for every $\varepsilon>0$ there is $y \in T$ with $d(x, y)<\varepsilon$; 
if $\bar{T}=S$, then $T$ is dense in $S$. A metric space $S$ is separable whenever $S$ possesses a dense and countable subclass $T$; by replacement, any such $T$ is a set even if $S$ is not.

A mapping $f: S \rightarrow \mathbb{R}$ on a metric space $S$ is a location in $S$ whenever

(i) $f(x) \geqslant|f(y)-d(x, y)|$ for all $x, y \in S$ and

(ii) $\inf _{x \in S} f(x)=0$.

All the locations in $S$ form the completion $\widehat{S}$ of $S$, which is a metric space with the metric

$$
d(f, g)=\inf _{x \in S}(f(x)+g(x)) .
$$

By assigning the location $f_{z}(x)=d(z, x)$ to every point $z \in S$, one can embed $S$ into $\widehat{S}$. This embedding is an isometry, because

$$
d(y, z)=\inf _{x \in S}(d(y, x)+d(z, x))=d\left(f_{y}, f_{z}\right) .
$$

We therefore may identify $S$ with its image in $\widehat{S}$; with this convention, $S$ is dense in $\widehat{S}$. A metric space $S$ is complete whenever $S=\widehat{S}$; as one might already expect, every completion is complete. For all this, see [21].

If $T \subseteq S$, then $\widehat{T}$ can be embedded isometrically into $\widehat{S}$ (for short, $\widehat{T} \subseteq$ $\widehat{S})$ by mapping each location $h$ on $T$ to the location $f_{h}$ on $S$ with $f_{h}(x)=$ $\lim _{h(y) \rightarrow 0} d(y, x)$. Note that this is a generalisation of the aforementioned construction: if $T=\{z\}$, then $\widehat{T}=\left\{h_{0}\right\}$ with $h_{0}(z)=0$, and $f_{h_{0}}=f_{z}$.

Lemma 5.1. If $T \subseteq S$, then $\bar{T} \subseteq \widehat{T}$; in particular, if $T$ is dense in $S$, then $\widehat{T}=\widehat{S}$.

Proof. Let $T \subseteq S$. If $z \in \bar{T}$, then $\inf _{x \in T} f_{z}(x)=\inf _{x \in T} d(z, x)=0$, and thus $f_{z} \in \widehat{T}$. This proves $\bar{T} \subseteq \widehat{T}$. As a consequence, if $\bar{T}=S$, then $S \subseteq \widehat{T}$ and thus $\widehat{S} \subseteq \widehat{\widehat{T}}$. Since $\widehat{T}$ is complete, this yields the missing inclusion $\widehat{S} \subseteq \widehat{T}$.

By condition (i), every location is nonnegative, so that condition (ii) amounts to requiring that for every rational $r>0$ there is $x \in S$ with $f(x)<r$; in particular, if $S$ is a set, then to be a location is a restricted property. Again by (i), $\widehat{S}$ is contained in the class $\mathscr{C}(S, \mathbb{R})$ of uniformly continuous functions; whence if $S$ and $\mathscr{C}(S, \mathbb{R})$ are sets, then so is $\widehat{S}$.

Also, if $S$ is a set, then to be uniformly continuous is a restricted property, so that $\mathscr{C}(S, \mathbb{R})$ is a set whenever so are $S$ and $\mathbb{R}^{S}$. In view of Corollary 4.2, we now have the following.

Proposition 5.2. By exponentiation and binary refinement, if $S$ is a set, then so is $\widehat{S}$.

The requirement of $S$ being a set is not needed in case, for example, $S$ is separable.

THEOREM 5.3. By exponentiation and binary refinement, if $S$ is a separable metric space, then $\widehat{S}$ is a set.

Proof. As $S$ is separable, there is a dense and countable subset $T$ of $S$, for which $\widehat{T}$ is a set by Proposition 5.2. By Lemma 5.1, $\widehat{S}$ equals $\widehat{T}$.

Although Theorem 5.3 holds no matter whether $S$ is a set, it is a set a posteriori if, in addition, $S$ is complete. 
Corollary 5.4. By exponentiation and binary refinement, every complete separable metric space is a set.

Both in Theorem 5.3 and Corollary 5.4, the hypotheses of exponentiation and of binary refinement were only needed to guarantee, using the assumption that $S$ is separable, the presence of a dense subset $T$ of $S$ for which $\mathscr{C}(T, \mathbb{R})$, or even $\mathbb{R}^{T}$, is a set. If a subset $T$ of this kind is known to exist anyway, one does not have to suppose those hypotheses.

On the first glance, it is surprising that $\widehat{S}$ can be a set even when $S$ is not: in general $\widehat{S}$ has much more elements than $S$. To be a set, however, seems to be not only a matter of (small) size, but also of cohesion. Perhaps the latter counts even more than the former: while $S$ may consist of relatively scattered points, $\widehat{S}$ appears to have enough elements to fill the gaps.

All this is of some relevance for formalising constructive analysis, in which the complete separable metric spaces play a predominant role. Though in an unexpected way, it also confirms Bishop's intuition to concentrate on separable metric spaces in constructive analysis. In [7, Appendix A], he even argued for his view of the concept of an nonseparable metric space as an instance of 'pseudogenerality' from the constructive perspective.

Related phenomena in the context of formal topology have been investigated by Curi $[9,10,11,12,13]$. For instance, he has shown that the formal points of certain types of formal topologies form a set, including the formal counterparts of $\mathbb{R}$ and of complete metric spaces. Using fullness, Aczel carried this over to CZF, where he generalised it [1]. Also, Curi has deduced ${ }^{3}$ the conclusion of Corollary 4.2 from Aczel's 'main lemma', whose proof is done with full fullness [1]. As compared with this route, the virtue of our proof is that binary refinement suffices.

Acknowledgements. A grant by JAIST enabled the second author to invite the first and third in March 2004, during which visit this research was initialised. Giovanni Curi and Albert Ziegler commented on a draft of this paper.

\section{REFERENCES}

[1] Peter Aczel, Aspects of general topology in constructive set theory, Annals of Pure and Applied Logic, to appear.

[2] — The type theoretic interpretation of constructive set theory, Logic Colloquium '77 (A. Macintyre, L. Pacholski, and J. Paris, editors), North-Holland, Amsterdam, 1978, pp. 55-66.

[3] - The type theoretic interpretation of constructive set theory: Choice principles, The L. E. J. Brouwer Centenary Symposium (A. S. Troelstra and D. van Dalen, editors), North-Holland, Amsterdam, 1982, pp. 1-40.

[4] - The type theoretic interpretation of constructive set theory: Inductive definitions, Logic, Methodology, and Philosophy of Science VII (R. B. Marcus, G. J. W. Dorn, and P. Weingartner, editors), North-Holland, Amsterdam, 1986, pp. 17-49.

[5] Peter Aczel and Chris Fox, Separation properties in constructive topology, From Sets and Types to Topology and Analysis (L. Crosilla and P. Schuster, editors), Oxford University Press, 2005, to appear.

[6] Peter Aczel and Michael Rathjen, Notes on constructive set theory, 2000/01, Institut MittagLeffler preprint 40.

[7] ERRETt Bishop, Foundations of Constructive Analysis, McGraw-Hill, New York, 1967.

[8] Errett Bishop and Douglas Bridges, Constructive Analysis, Springer, Berlin, Heidelberg, 1985.

\footnotetext{
${ }^{3}$ Communication by email from 4 October 2004.
} 
[9] GIOVANnI CURI, The points of (locally) compact regular formal topologies, Reuniting the Antipodes-Constructive and Nonstandard Views of the Continuum (Proceedings of the Symposion), 1999 (P. Schuster, U. Berger, and H. Osswald, editors), Synthese Library 306, Kluwer, Dordrecht, 2001, pp. 39-54.

[10] - Constructive metrisability in point-free topology, Theoretical Computer Science, vol. 305 (2003), pp. 85-109.

[11] , Geometry of observations, Ph.D. thesis, University of Siena, 2003.

[12] - On the collection of points of a formal space, Annals of Pure and Applied Logic, to appear.

[13] - Exact approximations to Stone-Čech compactification, Forthcoming.

[14] Robert Lubarsky, Independence results around constructive ZF, Annals of Pure and Applied Logic, vol. 132 (2005), pp. 209-225.

[15] Per Martin-LöF, An intuitionistic theory of types: Predicative part, Logic Colloquium '73 (H. E. Rose and J. C. Sheperdson, editors), North-Holland, Amsterdam, 1975, pp. 73-118.

[16] - Intuitionistic Type Theory, Notes by G. Sambin of a series of lectures given in Padua, June 1980, Bibliopolis, Napoli, 1984.

[17] Per Martin-Löf, An intuitionistic theory of types, Twenty-Five Years of Constructive Type Theory (G. Sambin and J. Smith, editors), Oxford University Press, 1998, pp. 127-172.

[18] John Myhill, Constructive set theory, this Journal, vol. 40 (1975), pp. 347-382.

[19] Sara Negri and Daniele Soravia, The continuum as a formal space, Archive for Mathematical Logic, vol. 38 (1999), pp. 423-447.

[20] Erik Palmgren, Constructive completions of ordered sets, groups and fields, Annals of Pure and Applied Logic, (2005), to appear.

[21] Fred Richman, The fundamental theorem of algebra: A constructive development without choice, Pacific Journal of Mathematics, vol. 196 (2000), pp. 213-230.

[22] Giovanni SAmbin, Some points in formal topology, Theoretical Computer Science, vol. 305 (2003), pp. $347-408$.

\author{
UNIVERSITÀ DI FIRENZE \\ DIPARTIMENTO DI FILOSOFIA \\ VIA BOLOGNESE 52, FIRENZE 50139, ITALY \\ E-mail: Laura.Crosilla@unifi.it
}

\title{
JAPAN ADVANCED INSTITUTE FOR SCIENCE AND TECHNOLOGY
}

SCHOOL OF INFORMATION SCIENCE

TATSUNOKUCHI, ISHIKAWA 923-1292, JAPAN

E-mail: ishihara@jaist.ac.jp

UNIVERSITÄT MÜNCHEN

MATHEMATISCHES INSTITUT

THERESIENSTR. 39, MÜNCHEN 80333, GERMANY

E-mail: Peter.Schuster@mathematik.uni-muenchen.de 